\title{
Asbestos fibre dimensions and lung cancer mortality among workers exposed to chrysotile
}

\author{
Dana Loomis, ${ }^{1}$ John Dement, ${ }^{2}$ David Richardson, ${ }^{3}$ Susanne Wolf ${ }^{3}$
}

- An additional table is published online only. To view this file please visit the journal online (http://oem.bmj.com).

${ }^{1}$ School of Community Health Sciences, University of Nevada, Reno, Nevada, USA

${ }^{2}$ Duke University Medical Center, Durham, North Carolina, USA

${ }^{3}$ School of Public Health, University of North Carolina, Chapel Hill, North Carolina, USA

\section{Correspondence to}

Professor Dana Loomis, School of Community Health

Sciences/MS-274, University of Nevada, Reno, NV 89557, USA; dploomis@unr.edu

Accepted 10 October 2009

Published Online First

5 November 2009

\section{ABSTRACT}

Objectives To estimate exposures to asbestos fibres of specific sizes among asbestos textile manufacturing workers exposed to chrysotile using data from transmission electron microscopy (TEM) and to evaluate the extent to which the risk of lung cancer varies with fibre length and diameter.

Methods 3803 workers employed for at least 1 day between 1 January 1950 and 31 December 1973 in any of three plants in North Carolina, USA that produced asbestos textile products and followed for vital status through 31 December 2003 were included. Historical exposures to asbestos fibres were estimated from work histories and 3578 industrial hygiene measurements taken in 1935-1986. Exposure-response relationships for lung cancer were examined within the cohort using Poisson regression.

Results Indicators of fibre length and diameter obtained by TEM were positively and significantly associated with increasing risk of lung cancer. Exposures to longer and thinner fibres tended to be most strongly associated with lung cancer, and models for these fibres fit the data best. Simultaneously modelling indicators of cumulative mean fibre length and diameter yielded a positive coefficient for fibre length and a negative coefficient for fibre diameter. Conclusions The results support the hypothesis that the risk of lung cancer among workers exposed to chrysotile asbestos increases with exposure to longer fibres. More research is needed to improve the characterisation of exposures by fibre size and number and to analyse the associated risks in a variety of industries and populations.

The occurrence of cancer among workers exposed to asbestos has been studied extensively and whilst the carcinogenicity of asbestos is established, there is significant variation in risk both among and within the industries using asbestos. ${ }^{12}$ It has been suggested that this might be explained by variations in the distribution of asbestos fibre sizes and shapes in addition to differences in the types of asbestos. $^{2-5}$ Fibre size distributions have been shown to vary between industries and processes, ${ }^{3} 6$ and data from experimental studies generally suggest that long, thin fibres may have greater carcinogenic potency than shorter, wider fibres. ${ }^{7-9}$

The hypothesis that the risk of cancer from exposure to asbestos may depend on fibre size is of scientific and regulatory interest, but relevant epidemiological data are limited because size distributions of all airborne fibres cannot be determined by the approaches usually used to measure asbestos concentrations in air. Since the 1960s, most exposure measurements have been made using phase-contrast light miscroscopy (PCM) to count fibres retained on filters according to stan-

\section{What this paper adds}

- Animal evidence suggests that the carcinogenicity of asbestos fibres increases with their length, but only limited human data are available to test this hypothesis.

- This study found that exposure to longer fibres was associated with higher rates of lung cancer among workers historically exposed to chrysotile asbestos.

- Assessments of exposure to asbestos should account for fibre sizes, as well as numbers, and the associated cancer risks should be examined in future epidemiological studies.

dard protocols that yield fibre-number concentrations. While PCM methods remain the standard procedure for regulatory compliance, the counts exclude significant numbers of fibres that may be biologically relevant. The standard protocol includes only fibres $>5 \mu \mathrm{m}$ long with length:width aspect ratios $\geq 3$ and fibres less than about $0.25 \mu \mathrm{m}$ in diameter are usually omitted because they are too small to resolve with most light microscopes. Fibres shorter than $5 \mu \mathrm{m}$ account for the majority of airborne fibres in several industries. ${ }^{3} 510$

Use of more recently developed methods using electron microscopy allows all fibres to be counted and classified, and may offer an opportunity to significantly improve the quantification of asbestos exposures and refine risk estimates. The objectives of this study were to use transmission electron microscopy (TEM) to estimate exposures to asbestos fibres of specific sizes among a cohort of asbestos textile manufacturing workers, and to evaluate the extent to which the risk of lung cancer varies with fibre length and diameter.

\section{METHODS}

\section{Study sites and population}

The facilities and workers included in the study were described in detail in an earlier paper. ${ }^{10}$ Briefly, the study includes 2419 men and 1384 women (total $\mathrm{N}=3803$ ) employed in any of three asbestos textile plants in North Carolina, USA, for at least 1 day between 1 January 1950 and 31 December 1973. All three plants engaged in the full process of textile production, which involved conversion of raw asbestos and cotton fibres into yarn and woven materials. Two of the plants were producing asbestos textiles in the 1920s and the third began in the 1940s; one plant closed in 1970, while the others continued to produce asbestos products as late as the 1990s. Records indicate that only chrysotile asbestos was oem.bmi.com/site/about/ unlocked.xhtml 
used with the exception of a small insulation operation in one plant, where limited amounts of amosite were carded, twisted and woven between 1963 and 1976.

The study population was enumerated from several sources ${ }^{10}$ and their vital status was ascertained through 31 December 2003. Causes of death, including underlying cause, immediate causes and other significant conditions, were coded to the International Classification of Diseases (ICD) in effect at the time of death. Procedures involving human subjects were approved by the Institutional Review Boards of the University of North Carolina, Chapel Hill and the University of Nevada, Reno.

For the analyses reported here, workers who did not have a complete occupational history specifying at least the department for all jobs and those who had ever been employed in nonproduction areas were excluded because their exposures could not be estimated. The population included in this study is thus identical to that for which exposure-response findings were reported by Loomis et $a l^{10}$ and includes 124029 person-years of follow-up, 1681 total deaths and 181 deaths from lung cancer.

\section{Exposure assessment}

The assessment of exposure for this study is described briefly here and in more detail in previous publications. ${ }^{51011}$ The first phase of exposure assessment focused on estimating asbestos fibre concentrations according to the standard PCM method, which was used in the study plants from 1964. In total, 3420 historical industrial hygiene measurements covering the period 1935-1986 were available for this purpose. Measurements taken before 1964 used the impinger method, and these were converted to PCM units as described by Dement et al. ${ }^{11}$ PCMequivalent fibre concentrations specific to plant, department, job and time period were then estimated by fitting multivariable linear mixed models to the data. The fitted values obtained from the model were used to construct a job-exposure matrix of estimated PCM fibre concentrations by plant, department, job and year.

In the second phase of exposure assessment, fibre concentrations were estimated according to fibre length and diameter. TEM was used to estimate the distribution of fibres for each combination of plant and department in categories defined by diameter (four categories) and length (six categories). A stratified random sample of 77 historical dust samples captured on membrane filters was selected from among 333 samples available from industrial hygiene studies the US Public Health Service conducted in the study plants during 1964-1971 and now archived at the National Institute for Occupational Safety and Health. The TEM fibre-counting protocol was based on the ISO direct-transfer method ${ }^{12}$ and data reduction and derivation of size-specific exposure estimates followed the procedure described by Dement et al. ${ }^{511}$ A total of 22776 fibres or fibre bundles were counted and sized.

The bivariate fibre diameter/length distributions from TEM were then used to estimate size-specific fibre exposures using a method proposed by Quinn et al, ${ }^{13}$ in which standard fibre concentration measures determined by PCM are adjusted to size-specific fibre concentrations using proportions from bivariate fibre size distributions. Adjustment factors were developed for each length-diameter category and applied to the matrix of plant-, department-, job- and time-specific PCM fibre concentrations to produce fibre size-specific estimates of exposure. ${ }^{11}$ Biologically based indices of fibre size proposed by Lippmann ${ }^{8}$ and Berman et al $^{14}$ were also computed.

Estimated exposures to fibres of different dimensions were linked to workers' occupational histories for assignment of individual cumulative exposure. Work histories and exposure estimates were coded using the same categories. Cumulative exposure to each class of fibres was estimated in fibre-years $/ \mathrm{ml}$ $(\mathrm{f}-\mathrm{y} / \mathrm{ml})$.

Because textile production generates fibres with wide length and diameter ranges, individual workers were exposed to fibres of multiple sizes simultaneously and throughout their careers. Consequently, indicators of exposure based on categories of fibre length and diameter tend to be highly correlated. To reduce collinearity and allow the effects of fibre length and diameter to be modelled simultaneously, we developed indicators to represent the mean length and diameter of the fibres to which workers were exposed. Cumulative mean fibre length was estimated by the quantity $\sum \bar{L}_{i} c_{i} d_{i} / \sum c_{i}$, where $\bar{L}_{i}$ is the mean length of fibres in length-diameter category $i$, and $c_{i}$ and $d_{i}$ are the concentration of fibres and the duration of employment in category $i$, respectively. Cumulative mean fibre diameter was estimated similarly by substituting the mean diameter of fibres in length-diameter category $i$ for $L_{i}^{-}$. Both indicators have units of $\mu \mathrm{m}$-years $(\mu \mathrm{m}-\mathrm{y})$.

\section{Data analysis}

Exposure-response analyses were based on 181 deaths from lung cancer. The number of deaths from mesothelioma $(n=8)$ was insufficient for analysis. Lung cancer mortality rates were modelled using Poisson regression following the approach employed in previous internal analyses of this cohort. ${ }^{10}$ The association of lung cancer with indicators of fibre exposure was estimated as $e^{\beta X}$, where $\beta$ is a regression coefficient for exposure $X$, and $95 \%$ confidence intervals (CI) were estimated from the standard error of $\beta$ using a normal approximation. ${ }^{15}$ The overall fit of the models was evaluated by the Akaike Information Criterion (AIC), which uses a penalty for the number of terms to allow the fit of non-nested models to be directly compared. ${ }^{16}$ The contribution of the exposure term was evaluated by likelihood ratio (LR) $\chi^{2}$ test. The ungrouped form of Poisson regression, equivalent to the discrete-time proportional hazards model, was used to allow predictors to be entered in continuous or categorical form in the same model. ${ }^{17}$ Deaths with any mention of lung cancer on the death certificate were included in the analysis.

For consistency with previous analyses, the final model included age entered with categories $<60,60-69,70-79$ and $80+$ years, sex, race with categories of white and other or unknown, calendar time with categories for each of the decades 1950-2000, and birth cohort with categories of $<1920,1920-1939$ and 1940 or later. Age, calendar time and exposure were all time-related variables, and exposure estimates were lagged by 10 years to account for latency. A longer latency period is also biologically plausible, but previous analyses of this cohort showed that lags longer than 10 years had little effect on the regression coefficients or on model fit. ${ }^{10}$ Indicators of fibre exposure were entered as continuous variables using both linear terms and penalised spline functions, which allow exposure-response relationships to take smooth, non-linear forms. ${ }^{18}$ Models in which exposure was entered as a spline function suggested a linear response and did not result in improved fit, so only results for standard linear terms are reported here. Regression analyses were carried out using $\mathrm{R}$ version 2.7.2 for Mac OS X. ${ }^{19}$

Comparisons of models for different fibre-size indicators were based on model goodness of fit and the likelihood ratio for the exposure term, as well as on the magnitude and precision of the regression coefficients. Although rate ratios or regression coefficients are normally of primary interest, in this case direct comparison of these measures is complicated because the number of fibres varies among categories while the number of deaths is 
fixed, so coefficients (or rate ratios) for categories with fewer fibres will be larger given equal cancer rates. To facilitate comparisons among fibre-size indices with different distributions, we scaled the regression coefficients by the IOR for each indicator.

\section{RESULTS}

\section{Fibre exposures}

As expected, total cumulative exposure to fibres among the 3803 workers included in the cohort was far greater when estimated by TEM (mean $989.4 \mathrm{f}-\mathrm{y} / \mathrm{ml}$ lagged 10 years) compared to the estimate obtained by PCM methods (mean $59.2 \mathrm{f}-\mathrm{y} / \mathrm{ml}$ lagged 10 years). When cumulative exposure was estimated by fibresize category, exposures were highest for the smallest fibres $<0.25 \mu \mathrm{m}$ long and $\leq 1.5 \mu \mathrm{m}$ long and tended to decrease with both fibre length and fibre width. Detailed descriptive data on cumulative exposure are given in online supplementary table S1.

\section{Lung cancer risk and TEM fibres}

Cumulative exposure to all fibres counted by TEM was significantly associated with lung cancer risk (table 1). The model for total TEM fibres did not fit as well as a model using exposure estimated by PCM, however, and the likelihood ratio was larger for PCM than for all TEM fibres (LR 9.6, $p=0.002$ for PCM vs LR 7.6, $\mathrm{p}=0.006$ for TEM fibres). The model for TEM fibres $>5 \mu \mathrm{m}$ long, which correspond most closely to PCM estimates, fit the data better than models for other TEM exposure indicators and the exposure term was more highly significant $(p=0.004)$. The strength of the association with lung cancer was similar for all TEM and PCM exposure indicators, with risk increasing about $3 \%$ for an increase in exposure equivalent to $1 \mathrm{IOR}$.

\section{Lung cancer risk by fibre size category}

Cumulative exposure to fibres in every length and diameter category was associated with lung cancer risk when each dimension was considered separately (table 2). Goodness of fit and strength of association with lung cancer tended to increase in models for fibres $>10 \mu \mathrm{m}$ in length, but similar results were obtained for very short fibres $\leq 1.5 \mu \mathrm{m}$ long and for fibres $>3 \mu \mathrm{m}$ in diameter. Both the best model fit and the strongest associations with lung cancer were achieved for cumulative exposure to fibres $20-40 \mu \mathrm{m}$ in length (table 2). These models fit the data better than models for PCM fibres and the exposure terms were more strongly associated with lung cancer risk (cf table 1).

When fibre length and diameter were considered in combination, exposures to several categories of shorter, larger-diameter fibres were not significantly associated with lung cancer, while stronger, statistically significant associations were observed for longer and thinner fibres, particularly those $>20 \mu \mathrm{m}$ long and $0.25-<1.0 \mu \mathrm{m}$ in diameter (table 2). Models for fibres $>20 \mu \mathrm{m}$

Table 1 Model goodness of fit and association of lung cancer risk with indicators of cumulative exposure to asbestos fibres, estimated by Poisson regression with adjustment for age, sex, race, calendar time and birth cohort

\begin{tabular}{lllllll}
\hline Exposure indicator $(\mathbf{f}-\mathbf{y} / \mathbf{m l})$ & $\beta$ & SE $(\beta)$ & $\Delta$ & LR & $\mathbf{p}$ & AIC \\
\hline PCM fibres & 0.00101 & 0.00028 & 0.0333 & 9.6 & 0.002 & 2343.1 \\
Total TEM fibres & 0.00005 & 0.00002 & 0.0310 & 7.6 & 0.006 & 2345.1 \\
TEM fibres $\geq 5 \mu \mathrm{m}$ & 0.00039 & 0.00012 & 0.0312 & 8.5 & 0.004 & 2344.2 \\
TEM fibres $<5 \mu \mathrm{m}$ & 0.00006 & 0.00002 & 0.0297 & 7.4 & 0.010 & 2345.3
\end{tabular}

AIC, Akaike Information Criterion (smaller values indicate better fit); $\beta$ and $\mathrm{SE}$, regression coefficient and associated standard error; $\Delta$, change in lung cancer risk for increment in exposure equal to 1 IQR; LR, likelihood ratio test statistic (equivalent to $\chi^{2}$ with 1 degree of freedom) and associated $\mathrm{p}$ value; PCM, phase-contrast light miscroscopy; TEM,

transmission electron microscopy.
Table 2 Model goodness of fit and association of lung cancer risk with cumulative exposure to asbestos fibres by size category, estimated by Poisson regression with adjustment for age, sex, race, calendar time and birth cohort

\begin{tabular}{|c|c|c|c|c|c|}
\hline \multirow[b]{2}{*}{ Length $(\mu \mathrm{m})$} & \multicolumn{5}{|c|}{ Diameter $(\mu \mathrm{m})$} \\
\hline & $<0.25$ & $0.25-1.0$ & $1.0-3.0$ & $>3.0$ & All \\
\hline \multicolumn{6}{|l|}{$\leq 1.5$} \\
\hline AIC & 2343.9 & 2350.2 & - & - & 2344.1 \\
\hline$\Delta$ & 0.0328 & 0.0149 & & & 0.0323 \\
\hline LR & 8.8 & 2.5 & & & 8.6 \\
\hline \multicolumn{6}{|l|}{$1.5-5$} \\
\hline AIC & 2347.5 & 2349.1 & 2349.7 & - & 2347.8 \\
\hline$\Delta$ & 0.0229 & 0.0127 & 0.0133 & & 0.0156 \\
\hline LR & 5.2 & 3.6 & 3.0 & & 5.0 \\
\hline \multicolumn{6}{|l|}{$5-10$} \\
\hline AIC & 2346.4 & 2345.3 & 2347.4 & 2350.2 & 2345.7 \\
\hline$\Delta$ & 0.0210 & 0.0288 & 0.0157 & 0.0007 & 0.0250 \\
\hline LR & 6.3 & 7.4 & 5.3 & 2.5 & 7.0 \\
\hline \multicolumn{6}{|l|}{$10-20$} \\
\hline AIC & 2345.4 & 2343.9 & 2346.3 & 2349.3 & 2344.3 \\
\hline$\Delta$ & 0.0290 & 0.0327 & 0.0208 & 0.0082 & 0.0327 \\
\hline LR & 7.3 & 8.8 & 6.4 & 3.4 & 8.4 \\
\hline \multicolumn{6}{|l|}{$20-40$} \\
\hline AIC & 2342.6 & 2340.7 & 2344.1 & 2338.2 & 2340.0 \\
\hline$\Delta$ & 0.0337 & 0.0326 & 0.0203 & 0.0265 & 0.0371 \\
\hline LR & 10.1 & 12.1 & 8.6 & 14.5 & 12.7 \\
\hline \multicolumn{6}{|l|}{$>40$} \\
\hline AIC & 2344.1 & 2340.9 & 2346.9 & 2345.1 & 2341.8 \\
\hline$\Delta$ & 0.0367 & 0.0367 & 0.0225 & 0.0144 & 0.0367 \\
\hline LR & 8.6 & 11.8 & 5.9 & 7.6 & 10.9 \\
\hline \multicolumn{6}{|l|}{ All } \\
\hline AIC & 2344.9 & 2347.5 & 2346.4 & 2342.6 & 2345.1 \\
\hline$\Delta$ & 0.0310 & 0.0202 & 0.0242 & 0.0256 & 0.0310 \\
\hline LR & 7.8 & 5.2 & 6.3 & 10.1 & 7.6 \\
\hline
\end{tabular}

Structures $\leq 1.5 \mu \mathrm{m}$ long and $>1 \mu \mathrm{m}$ wide and $1.5-5 \mu \mathrm{m}$ wide and $>3 \mu \mathrm{m}$ wide do not meet the definition of a fibre and were not counted.

AIC, Akaike Information Criterion (smaller values indicate better fit); $\Delta$, change in lung cancer risk for increment in exposure equal to 1 IQR; LR, likelihood ratio (equivalent to $\chi^{2}$ with 1 degree of freedom).

long fit the data best, but in contrast to the general pattern favouring thinner fibres, the best fit for any single fibre lengthdiameter category was obtained for fibres $20-40 \mu \mathrm{m}$ long and $>3 \mu \mathrm{m}$ in diameter. The model for the smallest fibres $\leq 1.5 \mu \mathrm{m}$ long and $<0.25 \mu \mathrm{m}$ in diameter was also an exception to the overall pattern, with both better fit and a stronger association with lung cancer compared to adjacent categories (table 2). Models that included terms for multiple length-diameter categories simultaneously failed to converge, probably because of collinearity.

\section{Alternative indicators of fibre exposure}

The biologically based exposure indicators we examined were significantly associated with increasing lung cancer risk (table 3). The best fit was obtained with an index based on Lippmann's suggestion that fibres $>10 \mu \mathrm{m}$ long and $0.3-0.8 \mu \mathrm{m}$ thick should be most relevant to lung cancer risk. ${ }^{8}$ The change in risk per IOR was modestly greater, however, for the index proposed by Berman which assigns empirical weights for relative potency to fibres in the categories $<0.3 \mu \mathrm{m}$ in diameter and 5-40 $\mu \mathrm{m}$ long, $<0.3 \mu \mathrm{m}$ in diameter and $>40 \mu \mathrm{m}$ long, and $>3 \mu \mathrm{m}$ in diameter and $>40 \mu \mathrm{m}$ long. ${ }^{14}$ We also considered exposure to long, thin fibres $<0.25 \mu \mathrm{m}$ in diameter and $\geq 10 \mu \mathrm{m}$ long, which are similar to the size range hypothesised by Stanton to be most relevant for carcinogenesis. ${ }^{7}$ This index of exposure was also associated with lung cancer, but less strongly than others (table 3). 
Table 3 Model goodness of fit and association of lung cancer risk with alternative indicators of cumulative exposure to asbestos fibres, estimated by Poisson regression with adjustment for age, sex, race, calendar time and birth cohort

\begin{tabular}{|c|c|c|c|c|c|c|}
\hline Exposure indicator & $\beta$ & SE $(\beta)$ & $\Delta$ & LR (df) & $\mathbf{p}$ & AIC \\
\hline \multicolumn{7}{|l|}{ Biologically-based indices* } \\
\hline $\begin{array}{l}\text { Modified Lippmann Index } \\
(0.25 \leq D \leq 1 \mu \mathrm{m} \text { and } \mathrm{L} \geq 10 \mu \mathrm{m}) \dagger\end{array}$ & 0.00283 & 0.00073 & 0.0279 & $11.1(1)$ & 0.001 & 2341.6 \\
\hline TEM fibres $(\mathrm{D}<0.25 \mu \mathrm{m}$ and $\mathrm{L} \geq 10 \mu \mathrm{m})$ & 0.00171 & 0.00054 & 0.0321 & $8.3(1)$ & 0.004 & 2344.4 \\
\hline \multicolumn{7}{|l|}{ Ad hoc indices $(\mu m-y)$} \\
\hline Mean fibre length & 0.00869 & 0.00257 & 0.0757 & $9.7(1)$ & 0.002 & 2343.1 \\
\hline Mean fibre diameter & -0.18835 & 0.42987 & -0.0735 & & & \\
\hline
\end{tabular}

*Units are $f-y / m l$. All of the indices also include the criterion that the aspect ratio (length:diameter) is at least 3:1.

†Lippmann proposed minimum diameters of $0.15 \mu \mathrm{m}$ or $0.3 \mu \mathrm{m}$, but we used a cut-off of $\geq 0.25 \mu \mathrm{m}$ since that was the closest category in our TEM protocol.

AIC, Akaike Information Criterion (smaller values indicate better fit); $\beta$ and SE, regression coefficient and associated standard error; $\Delta$, change in lung cancer risk for increment in exposure equal to 1 IQR; D, diameter; L, length; LR (df), likelihood ratio test statistic (equivalent to $\chi^{2}$ ) with degree of freedom (df) and associated $p$ value; TEM, transmission electron microscopy.

The indicators of cumulative mean fibre length and diameter we developed for this study were positively and significantly associated with lung cancer risk; the model for mean fibre length provided the best fit to the data (table 3). When terms for mean fibre length and diameter were entered simultaneously, the coefficient for length remained positive, while that for diameter became negative, consistent with increasing risk associated with longer, thinner fibres (table 3). A term for the interaction of fibre length and diameter was not statistically significant, but the coefficient was negative $(\beta=-0.00011, p=0.92)$, consistent with the effect of greater fibre length diminishing with increasing fibre diameter. Interactions of total TEM fibres with fibre length or fibre diameter were also non-significant $(p=0.70$ and $p=0.72$, respectively).

\section{DISCUSSION}

Indicators of asbestos fibre length and diameter obtained by analysing historical dust samples via TEM were positively and significantly associated with increasing risk of lung cancer in a cohort of asbestos textile workers exposed to commercial chrysotile. The strength of association varied modestly by fibre length and diameter, but in general models for exposure to longer fibres fit the data best and indicated the strongest associations with lung cancer. Findings for fibre diameter were less consistent, but simultaneously modelling indicators of cumulative mean fibre length and diameter yielded a positive coefficient for fibre length and a negative coefficient for fibre diameter, as would be expected if risk increased with greater fibre length and smaller fibre diameter.

The effect of asbestos fibre dimensions on lung cancer risk in humans has also been examined directly in a study of workers employed in a similar asbestos textile plant in South Carolina, USA. $^{20} 21$ Fibre exposures were assessed using the same methods and TEM protocols we used, and the fibre-size distributions were similar. ${ }^{511}$ The major findings from analysis of the relationship of lung cancer to fibre size in the South Carolina plant were similar to those we present here. All of the TEM-based indicators of fibre length and diameter considered in that study were associated with lung cancer, with the strongest associations observed for long fibres (length $>10 \mu \mathrm{m}$ ) and very thin fibres (diameter $<0.25 \mu \mathrm{m}$ ). ${ }^{20}$ In contrast to our findings, however, all TEM-based exposure indicators for the South Carolina cohort were more strongly associated with lung cancer than estimates based on PCM measurements. ${ }^{20}$

All other epidemiological studies to date have used exposure estimates based on standard PCM fibre-counting methods. A meta-analysis of 20 such studies by Berman and Crump ${ }^{22}$ examined the effects of asbestos fibre type and size on the risk of cancer using surrogate estimates of fibre size for 19 cohorts and published fibre-size distributions for the South Carolina asbestos textile cohort. The results suggest that lung cancer risk is associated most strongly with exposure to fibres longer than $10 \mu \mathrm{m}$, but no notable variation in risk with fibre diameter was reported and exposure indicators based on fibre-size estimates fit the data only marginally better than estimates of PCM-equivalent fibres. ${ }^{22}$ The authors concluded that their analysis failed to fully explain the differences in cancer risk among asbestos-using industries. Nevertheless, their finding of stronger associations with longer fibres is consistent with our findings for North Carolina asbestos textile workers and with those reported for South Carolina asbestos textile workers. ${ }^{20}$

Our major findings are consistent with several experimentally and theoretically based expectations about the relative carcinogenicity of fibres according to their length and diameter. Lippmann ${ }^{8}$ concluded from a review of findings of experiments with animals exposed to asbestos by inhalation that long fibres $(>10 \mu \mathrm{m})$ are likely to be most carcinogenic to the lung. We found that these long fibres were consistently associated with lung cancer in North Carolina asbestos textile workers. Lippmann also proposed that, while all fibres $>0.15 \mu \mathrm{m}$ in diameter may be relevant to tumour induction, those $0.3-0.8 \mu \mathrm{m}$ in diameter are most likely to be retained in the lung and therefore to be associated with higher risk. We could not evaluate fibres in these specific diameter ranges, but we found that long fibres in the nearest diameter class $(0.25-1.0 \mu \mathrm{m})$ were more strongly associated with lung cancer risk than thinner or thicker fibres the same length. Berman et al ${ }^{14}$ concluded from a re-analysis of previous rodent inhalation experiments that fibres $>5 \mu \mathrm{m}$ long and $<0.3 \mu \mathrm{m}$ thick appeared to predict lung tumour risk most strongly, with the possibility of additional contributions from very long fibres $>40 \mu \mathrm{m}$ in length and very thick fibre bundles and structures $>5 \mu \mathrm{m}$ in diameter. Our findings for exposures to fibres $<0.25 \mu \mathrm{m}$ in diameter and $>5 \mu \mathrm{m}$ long are consistent with these observations. We also found relatively strong associations for thin fibres $>40 \mu \mathrm{m}$ long, as well as for thick fibres $>10 \mu \mathrm{m}$ long. Very thick fibres are usually considered to have low pathogenic potential because of their low rates of deposition and retention. ${ }^{8}$ However, long, thick chrysotile structures in airborne dust may disintegrate into thinner fibrils, making additional long fibres available to the lung. ${ }^{8} 14$

Several other potential indices for biologically active fibres have been reviewed by Quinn et al ${ }^{13}$ and Dement et al. ${ }^{5}$ We did not conduct analyses with these indices because the length and diameter cut-points and aspect ratios they require differ from 
our TEM protocol. The Hypothetically Active Fibre (HAF) index developed by Quinn ${ }^{23}$ also requires data on fibre persistence in the lung. Hypotheses advanced by Stanton ${ }^{7}$ and by Pott ${ }^{9}$ about the aetiological importance of specific fibre-size ranges were based on studies of pleural toxicity in animals exposed to fibres by implantation or injection, but the fibre size ranges that are relevant to inhaled fibres and lung cancer are likely to be different. ${ }^{8}$

The role of the smallest fibres less than $1.5 \mu \mathrm{m}$ long and $0.25 \mu \mathrm{m}$ in diameter requires further investigation. It has been hypothesised on toxicological grounds that such short, thin fibres may not have a role in the genesis of lung cancer. ${ }^{7} 81424$ We found that short, thin fibres were the majority of those counted by TEM, and exposure to them was associated with lung cancer. However, we cannot yet determine whether the association of these fibres with lung cancer is a spurious effect due to correlations among fibre-size categories or evidence that small fibres do play a role in carcinogenesis.

The strengths of the study include the large size of the cohort, the long follow-up period, the high proportion of workers who were successfully traced and the availability of extensive historical information on exposures. The epidemiological data have several limitations, which have been discussed previously. ${ }^{10}$ Briefly, these include: insufficient data to allow for control of smoking; occupational histories specifying only the plant and department, but not the job title, for about a quarter of the cohort; and the effects of a medical surveillance program that terminated exposures of atrisk workers, possibly attenuating the association of lung cancer with cumulative asbestos exposure.

The current analysis of fibre-dimension data obtained by TEM has further limitations. While archived dust samples were analysed for every combination of plant and department and a reasonably large number of structures were counted for each sample, these samples were available only for the years 1964-1971. Production processes and equipment did not change markedly during the years of the study. It is reasonable to assume that fibre-size distributions were stable throughout the period, but we have no data to test this assumption.

In addition, we did not have the resources to count large numbers of samples or to estimate exposures for specific jobs within departments. Additional uncertainty arises from the potential variability of the proportion of fibres in each lengthdiameter category and of the adjustment factors used to estimate fibre-size specific exposure from PCM measurements, neither of which was accounted for in the epidemiological analysis. Random measurement error arising from the small number of samples and lack of fine detail in the exposure assessment may have led to reduced power and attenuation of exposure-disease associations. ${ }^{25}$ These uncertainties may also explain the somewhat poorer fit of models for some TEM fibres compared to PCM fibres. Rapid, low-cost methods for determining size distributions in large numbers of samples will be needed for fibre-size analysis to be adopted as a practical tool for hygiene practice.

Finally, workers were exposed to fibres of a wide range of lengths and diameters, but strong correlations among fibre-size metrics prevented modelling multiple fibre indicators simultaneously to search for evidence that specific fibre-size ranges have independent effects

In summary, the results of this study support the hypothesis that the risk of lung cancer among workers exposed to chrysotile asbestos increases with exposure to longer fibres and provide some evidence that those effects are most pronounced for long fibres between 0.25 and $1.0 \mu \mathrm{m}$ in diameter. There is still uncertainty about the relative carcinogenicity of specific fibresize fractions, however. Assessments of asbestos exposure should account for fibre size, as well as number, and more epidemiological research is needed to examine the variation of cancer risk with fibre size in a variety of industries and populations.

Acknowledgements We thank Eileen Gregory for assistance with data management and processing, and Minggen Lu for statistical advice.

Funding Support for this research was provided by grant R01-OH007803 from the National Institute for Occupational Safety and Health (USA).

Competing interests Dana Loomis is Editor and David Richardson is Associate Editor of Occupational and Environmental Medicine.

Ethics approval This study was conducted with the approval of the University of North Carolina, Chapel Hill and the University of Nevada, Reno.

Provenance and peer review Not commissioned; externally peer reviewed.

\section{REFERENCES}

1. Hodgson JT, Darnton A. The quantitative risks of mesothelioma and lung cancer in relation to asbestos exposure. Ann Occup Hyg 2000;44:565-601.

2. Berman DW, Crump KS. Update of potency factors for asbestos-related lung cancer and mesothelioma. Crit Rev Toxicol 2008;38:1-47.

3. Dement JM, Wallingford KM. Comparison of phase contrast and electron microscopic methods for evaluation of occupational asbestos exposures. Appl Occup Environ Hyg 1990;5:242-7.

4. Stayner LT, Dancovic DA, Lemen RA. Occupational exposure to chrysotile asbestos and cancer risk: a review of the amphibole hypothesis. Am J Public Health 1996;86:179-86

5. Dement JM, Kuempel ED, Zumwalde RD, et al. Development of a fibre size-specific job-exposure matrix for airborne asbestos fibres. Occup Environ Med 2008;65:605-12

6. Gibbs GW, Hwang CY. Dimensions of airborne asbestos fibres. Lyon, France: IARC Scientific Publications, 1980:69-78.

7. Stanton MF, Layard M, Tegeris A, et al. Relation of particle dimension to carcinogenicity in amphibole asbestoses and other fibrous minerals J Natl Cancer Inst 1981;67:965-75

8. Lippmann M. Asbestos exposure indices. Environ Res 1988;46:86-106

9. Pott F. [Fiber as a carcinogenic agent] (In German.). Zentralbl Bakteriol Mikrobiol Hyg B 1987:184:1-23.

10. Loomis D, Dement JM, Wolf SH, et al. Lung cancer mortality and fibre exposures among North Carolina asbestos textile workers. Occup Environ Med 2009;66:535-42

11. Dement J, Meyers D, Loomis D, et al. Estimates of historical exposures by phase contrast and transmission electron microscopy in North Carolina, USA asbestos textile plants. Occup Environ Med 2009;66:574-83.

12. International Standards Organisation. Ambient air-determination of asbestos fibres-direct transfer electron microscopy method. Geneva: International Standards Organisation, 1995.

13. Quinn MM, Smith TJ, Eisen EA, et al. Implications of different fiber measures for epidemiologic studies of man-made vitreous fibers. Am J Ind Med 2000;38:132-9.

14. Berman DW, Crump KS, Chatfield EJ, et al. The sizes, shapes, and mineralogy of asbestos structures that induce lung tumors or mesothelioma in AF/HAN rats following inhalation. Risk Anal 1995;15:181-95.

15. Frome EL. The analysis of rates using Poisson regression models. Biometrics 1983;39:665-74

16. McCullagh P, Nelder JA. Generalized linear models. 2nd edn. London: Chapman \& Hall, 1989.

17. Loomis D, Richardson D, Elliott L. Poisson regression analysis of ungrouped data. Occup Environ Med 2005;62:325-9.

18. Eilers PHC, Marx BD. Flexible smoothing with B-splines and penalties. Stat Sci 1996;11:89-121.

19. R Development Core Team. $R$ : A language and environment for statistical computing. Vienna, Austria: R Foundation for Statistical Computing, 2008.

20. Stayner L, Kuempel E, Gilbert S, et al. An epidemiological study oif the role of chrysotile asbetos fibre dimensions in determining respiratory disease risk in exposed workers. Occup Environ Med 2008;65:613-19.

21. Hein MJ, Stayner LT, Lehman E, et al. Follow-up study of chrysotile textile workers: cohort mortality and exposure-response. Occup Environ Med 2007:64:616-25.

22. Berman DW, Crump KS. A meta-analysis of asbestos-related cancer risk that addresses fiber size and mineral type. Crit Rev Toxicol 2008:38(Suppl 1):49-73.

23. Quinn MM, Smith TJ, Ellenbecker MJ, et al. Biologically based indices of exposure to fibres for use in epidemiology Occupational Hygiene 1996;3:103-11.

24. Stanton MF, Laynard M, Tegeris A, et al. Carcinogenicity of fibrous glass: pleural response in the rat in relation to fiber dimension. J Natl Cancer Inst 1977; 58:587-603.

25. Armstrong BG. Effect of measurement error on epidemiological studies of environmental and occupational exposures Occup Environ Med 1998;55:651-6. 